\title{
Physiologie humaine appliquée, 2nde édition
}

\author{
Claude Martin, Bruno Riou, Benô̂t Vallet. Editions Arnette, John Libbey Eurotext, \\ Paris, 2017. ISBN : 978-2-7184-1421-8
}

\author{
Athanase Courbe, MD
}

Received: 6 June 2017/Revised: 11 August 2017/Accepted: 11 August 2017/Published online: 15 August 2017

(c) Canadian Anesthesiologists' Society 2017

La physiologie, dont l'étymologie grecque signifie discours sur la nature, est la spécialité scientifique qui étudie le fonctionnement des organismes vivants. De bonnes connaissances et une bonne compréhension de la physiologie humaine, et de sa petite sœur qui en découle la physiopathologie, sont des outils indispensables aux médecins que nous sommes. Elles permettent une meilleure appréhension de la situation clinique de notre patient, et par conséquent une meilleure prise en charge de celui-ci, aboutissant idéalement à une amélioration de son pronostic.

Dans cette seconde édition de «Physiologie humaine appliquée » éditée par Arnette, les auteurs ont réuni de nombreuses équipes de médecins français, pour la plupart anesthésistes et intensivistes, afin d'offrir une revue actualisée des grandes fonctions physiologiques de l'être humain.

De façon intelligente, les grandes fonctions d'organe sont divisées en multiples chapitres (71 au total) relativement courts mais précis et bien détaillés pour ne pas « noyer » le lecteur dans trop d'explications à la fois et rendre le livre difficile à étudier.

Ainsi, chaque chapitre revoit, après un éventuel rappel anatomique lorsque c'est nécessaire, une fonction de l'organisme. De nombreuses illustrations, claires et bien pensées, aideront le lecteur à comprendre, intégrer et mémoriser les notions physiologiques expliquées. De plus, les nombreuses références bibliographiques permettront à ceux qui le souhaitent d'aller encore plus loin.

A. Courbe, MD $(\bowtie)$

Département d'anesthésiologie de l'Institut de Cardiologie de

Montréal, Montréal, QC, Canada

e-mail: athanase.courbe@gmail.com
Les auteurs des différents chapitres étant pour la plupart des cliniciens, en général spécialistes reconnus du sujet sur le plan médical, ils dépassent le champ de la physiologie et abordent des notions de physiopathologie, de monitorage clinique de la fonction physiologique étudiée, ou encore introduisent des notions sur le traitement d'une condition pathologique. Tous ces éléments ajoutent de l'intérêt pour le lecteur, et aident à une meilleure perception globale de la fonction physiologique considérée.

Dans un but d'exhaustivité, cette nouvelle édition comporte trois nouveaux chapitres que sont la dysfonction diaphragmatique (pathologie de plus en plus reconnue et étudiée, notamment dans le contexte des défaillances respiratoires postopératoires), la vision, ainsi qu'un chapitre audition et équilibre.

Aux chapitres de physiologie s'ajoutent également quelques chapitres traitant spécifiquement de physiopathologie. Ainsi sont traitées plusieurs situations engageant le pronostic vital et fréquemment rencontrées aux soins intensifs ou au bloc opératoire telles que les états de choc ou le syndrome de détresse respiratoire aiguë par exemple. Ces chapitres sont particulièrement utiles pour les médecins de notre spécialité puisqu'il s'agit de conditions pathologiques complexes et difficiles à gérer. Une bonne compréhension de leur physiopathologie aide à mieux cerner et prioriser notre approche thérapeutique.

De la même façon, la physiopathologie des principales techniques de suppléance d'organes employées en réanimation sont abordées. Le lecteur pourra ainsi mieux appréhender les enjeux de l'assistance circulatoire, la ventilation mécanique ou bien l'épuration extra-rénale qui font partie de l'arsenal thérapeutique quotidien de l'intensiviste. 
Par conséquent, ce livre est un excellent moyen d'apprentissage de la physiologie humaine pour l'étudiant en médecine, mais également pour un résident en formation dans le domaine de l'anesthésie ou des soins intensifs.

De plus, il constitue une référence de grande qualité dans le domaine de la physiologie pour le médecin anesthésiste ou intensiviste déjà formé qui souhaitent revoir des notions ou bien actualiser ses connaissances en fonction des récents progrès scientifiques.

\section{Conflit d'intérêt Aucun.}

Responsabilité éditoriale Cet article a été traité par Dr Étienne de Médicis, Rédacteur de Langue Française, Journal canadien d'anesthésie. 